\title{
SÉRIES ANUAIS DE TEMPERATURA MÁXIMA MÉDIA DO AR NO ESTADO DE SÃO PAULO: VARIAÇÕES E TENDÊNCIAS CLIMÁTICAS
}

\author{
GABRIEL CONSTANTINO BLAIN \\ Instituto Agronômico (IAC/APTA/SAA), Campinas, SP, Brasil \\ gabriel@iac.sp.gov.br \\ Recebido Dezembro 2008 - Aceito Agosto 2009
}

\begin{abstract}
RESUMO
O objetivo do trabalho foi analisar tendências e/ou variações climáticas em oito séries de temperatura máxima média anual (Tmax), do Estado de São Paulo. Por meio de testes paramétricos e nãoparamétricos foram detectadas, nas localidades de Pindorama e Ubatuba, consideráveis elevações nos dados de Tmax nos anos mais recentes dessas séries. Em contra partida, em Cordeirópolis, Mococa, Monte Alegre do Sul e Ribeirão Preto, não foram verificadas alterações temporais significativas (elevação ou queda) nas séries de Tmax. Nas localidades de Campinas e Piracicaba, foram observados marcantes deslocamentos à direita (elevação) das funções densidade de probabilidade associadas aos valores desse elemento meteorológico. Em Campinas, essa alteração ocorreu de forma mais intensa e temporalmente anterior à de Piracicaba. Dessa forma, apesar da maioria das séries apresentarem importantes variações em seus parâmetros estatísticos (variações climáticas), não foi possível estabelecer uma concomitância/significância na variabilidade temporal (elevação ou queda) nas oito séries de Tmax analisadas.
\end{abstract}

Palavras Chave: variabilidade temporal, funções densidade de probabilidade

\begin{abstract}
ANNUAL AVERAGE MAXIMUM AIR TEMPERATURE SERIES OF THE STATE OF SÃO PAULO, BRAZIL: CLIMATE VARIATIONS AND TRENDS.

The aim of the work was to detect climate trends or climate variations in eight annual average maximum air temperature series (Tmax) of the State of São Paulo. The study was based on parametric and nonparametric tests. In the Pindorama and Ubatuba series, positive trends in Tmax values were detected during the last 28/24 years. No significant trends were detected in the Cordeirópolis, Mococa, Monte Alegre do Sul, and Ribeirão Preto series. The probability density function associated with the series of Campinas and Piracicaba, shows significant shifts to the right. In the Campinas data series this shift on Tmax data distribution occurred 30 years (approximately) earlier than in the Tmax of Piracicaba data distribution. Thus, despite the majority of the data series have shown important variations on their statistical parameters, it was not possible to identify a temporal variability pattern on the eight Tmax analyzed series.
\end{abstract}

Keywords: temporal variability, probability density function

\section{INTRODUÇÃO}

Segundo o relatório do IPCC (2007), o termo mudança climática refere-se a alterações nos padrões climáticos, que podem ser identificadas por meio de alterações persistentes por longos períodos (da ordem de décadas) na média aritmética e/ou em outras medidas de sua variabilidade, independentes de suas causas. Apesar da atual preocupação da sociedade, estudos sobre esse tema não são recentes. A nota técnica ${ }^{\circ} 79$ (OMM, 1966), preocupa-se exclusivamente, com esse fenômeno, afirmando que o estudo de séries temporais meteorológicas demonstra claramente a inconstância do clima (climatic inconstancy). Para esse relatório, mudança climática (climatic change) é um termo genérico que engloba todas as formas de inconstância do clima, tais como tendências ou variações, independentemente de suas causas. Esses últimos dois conceitos são, segundo esse relatório, definidos por:

i) Tendência climática: caracterizada por uma elevação ou diminuição suave (smooth) e monotóna nos valores médios de uma série meteorológica. Não é, necessariamente, restrita a 
tendências lineares em função do tempo, mas deve conter apenas um máximo ou mínimo no ponto final da série;

ii) Variações climáticas: uma flutuação ou uma componente desta em escala de tempo suficientemente longa, capaz de resultar em inconstância nos parâmetros estatísticos relativos a períodos sucessivos de pelo menos 30 anos da variável meteorológica em questão.

Atualmente, entre os diversos esforços realizados sobre o tema de aquecimento global, destaca-se a detecção com base em métodos estatísticos de indícios de mudanças climáticas em longas séries meteorológicas. Alexander et al. (2006) analisando dados globais extremos de temperatura e precipitação, na escala diária, identificaram em $70 \%$ das regiões analisadas, significativa elevação nas temperaturas noturnas. Segundo esses autores, houve também detecção de elevação nos dados relativos à precipitação pluvial. Entretanto, essas últimas alterações apresentaram uma coerência espacial bastante inferior à observada nos dados de temperatura do ar. Haylock et al. (2006) analisando tendências em valores diários de precipitação na América do Sul (1960/2000), afirmam que o padrão de alteração observado nos valores extremos desse elemento meteorológico é, de modo geral, o mesmo para os totais anuais. Neste trabalho são verificadas mudanças para condições mais úmidas (changes to wetter conditions) na parte sul do Brasil. Marengo et al. (2007) observam, que no sudeste do Brasil, o total anual de precipitação parece não ter sofrido alteração nos últimos 50 anos.

Em estudos similares, voltados para a temperatura do ar, Vincent et al. (2005) observou tendências de elevação ligadas principalmente à temperatura noturna. Segundo esses autores, não houve, por meio dos índices empregados, detecção de significativas alterações (no consistent changes) nos dados de temperatura máxima do ar utilizados (1960/2000). Sansigolo (2008), em estudo relativo a intensificação dos valores extremos de precipitação, temperatura máxima e mínima do ar e velocidade do vento não detectou, com $95 \%$ de probabilidade, tendências climáticas significativas na série de Piracicaba. Dufek e Ambrizzi (2006) utilizando, entre outras, séries de temperatura máxima do ar pertencentes ao Instituto Agronômico (IAC/APTA/SAA) indicam que, no Estado de São Paulo há indícios de tendências para uma condição atmosférica mais quente. Os autores afirmam que particularmente para as regiões norte e central do Estado, essa elevação é associada a diminuição de dias frios nos anos de 1990 a 2002, sendo mais severa no período de inverno. Esse trabalho também afirma que uma análise sobre o crescimento urbano nas cidades e as mudanças no uso do solo, assim como seus impactos no clima local, ainda se faz necessária, a fim de descrever em detalhes as diferenças gerais obtidas entre os resultados de cada estação meteorológica.
Sob esse último aspecto, Blain et al. (2009), utilizando testes estatísticos paramétricos e não paramétricos, também indica consideráveis elevações nas séries anuais de temperatura mínima do Estado de São Paulo. Contudo, segundo esses autores, tanto a data inicial dessas elevações, quanto à significância das mesmas, são muito distintas entre as diferentes localidades analisadas indicando também a necessidade de isolar fatores de escala local, tais como urbanização, antes que estudos de zoneamento agrícola (e outros) sejam realizados com base nessas séries. Com isso, considerando as indicações de Blain et al. (2009) e de Dufek e Ambrizzi (2006), torna-se importante determinar a existência de elevações nas séries de temperatura máxima (Tmax) do Estado de São Paulo, indicando as datas aproximadas dessas possíveis alterações e suas respectivas significâncias estatísticas.

Dessa forma, o objetivo do trabalho foi analisar tendências e/ou variações nas séries de temperatura máxima média anual (Tmax) das localidades de Campinas, Cordeirópolis, Mococa, Monte Alegre do Sul, Piracicaba, Pindorama, Ribeirão Preto e Ubatuba, pertencentes ao Estado de São Paulo. Espera-se que esse trabalho contribua com futuros estudos relativos a esses postos em demais escalas temporais.

\section{MATERIAL E MÉTODOS}

É importante ressaltar que as séries relativas às localidades de Pindorama e Ubatuba, não são capazes de gerar duas amostras com pelo menos 30 anos (período mínimo exigido para a condução de caracterização climática). Dessa forma, sob o ponto de vista de rigor científico, análises de mudanças climáticas, especialmente variações climáticas, conduzidas nessas séries, carecem de robustez. Entretanto, optou-se por utilizá-las no estudo, ainda que seus resultados devam ser vistos com ressalvas. A caracterização estatística das séries de Tmax foi inicialmente realizada por meio do ajuste dessas distribuições empíricas à distribuição normal (DN), utilizando-se todo o período disponível (e, portanto diferentes) em cada localidade. A não adoção de um período comum para a condução desse ajuste é baseada no fato de que o maior período único, possível de ser adotado, é limitado pela menor série disponível (Ubatuba-SP), acarretando, com isso, em grande perda de dados (informações) nas demais regiões. $\mathrm{O}$ ajuste das distribuições empíricas à DN foi avaliado por meio do teste Kolmogorav-Smirnov (KS). São ilustradas na Figura 1 as localidades utilizadas no estudo.

As análises estatísticas empregadas foram baseadas em métodos paramétricos e não paramétricos. Nesse ponto, é interessante ressaltar Dale (1968), o qual afirma que o fato de um evento não ser registrado em uma amostra, probabilidade empírica igual à zero, não significa que ele não esteja ou não estará contido na população. Nesses casos, a distribuição 
empírica não refletirá todas as possíveis sucessões dos tipos de tempo locais, fazendo-se necessário o uso de distribuições teóricas paramétricas. Sob esse mesmo aspecto, Sansigolo e Nery (2000) afirmam que quando bem ajustados, os modelos teóricos resultam em maior embasamento estatístico da descrição climática, sendo importantes tanto em curtas quanto em longas séries.

Dessa forma, pode-se inferir que o primeiro passo em análises de alterações climáticas, seria o ajuste de cada uma das amostras a uma distribuição teórica conhecida. A análise de possíveis variações ou tendências climáticas seria realizada por meio do estudo dos parâmetros das distribuições relativas a essas amostras, diminuindo, com isso, a probabilidade de que as diferenças entre esses dois sub-períodos, sejam atribuídas a falhas na descrição climática da região. Em contra partida, é também interessante ressaltar Frei e Schar (2000), os quais afirmam que análises de tendências em longas séries meteorológicas confrontam-se com a dificuldade fundamental de que as variações estocásticas, presentes em qualquer série meteorológica, limitam a acurácia com a qual possíveis tendências podem ser detectadas. De acordo com esses autores, dois tipos de erro podem ocorrer: a) indicação de tendências climáticas, quando há apenas flutuações essencialmente controladas pelas variações estocásticas (erro tipo I); b) uma real tendência climática não é identificada por estar "encoberta" por flutuações estocásticas de pequenas escalas temporais (erro tipo II). Esses tipos de erro estão presentes em qualquer tipo de análise de mudanças climáticas, incluindo-se os relativos a valores médios (Frei e Schar, 2000). No presente caso, apesar do "amaciamento" causado pelo ajuste das distribuições empíricas à DN diminuir a probabilidade do erro tipo I aumenta, obviamente, a do tipo II. Com isso, torna-se também interessante analisar possíveis variações e tendências climáticas considerando-se testes não paramétricos.

\section{1 Métodos Paramétricos}

A disponibilidade de dados relativos às localidades de Campinas e Piracicaba, permitiu à condução dos testes paramétricos $\mathrm{t}$ (bi-caudal) e $\mathrm{F}$, conforme descritos na nota técnica da OMM (1966) e em Wilks (2006), em três sub-períodos iguais de 30 anos - 1918/1947, 1948/1977 e 1978/2007. Assim como indicado pela OMM (1966), adotou-se o nível de significância de $5 \%$ para a realização desses testes. A mesma análise foi aplicada às demais localidades utilizando-se, entretanto, apenas duas amostras em cada localidade (cada série foi dividida em dois sub-períodos com o mesmo número de anos). $\mathrm{O}$ ajuste de cada sub-período à DN foi também avaliado pelo KS.

O teste da razão da verossimilhança $\left(\Lambda^{*}\right)$ é indicado por Schickedanz e Krause (1969) e Wilks (2006), como uma

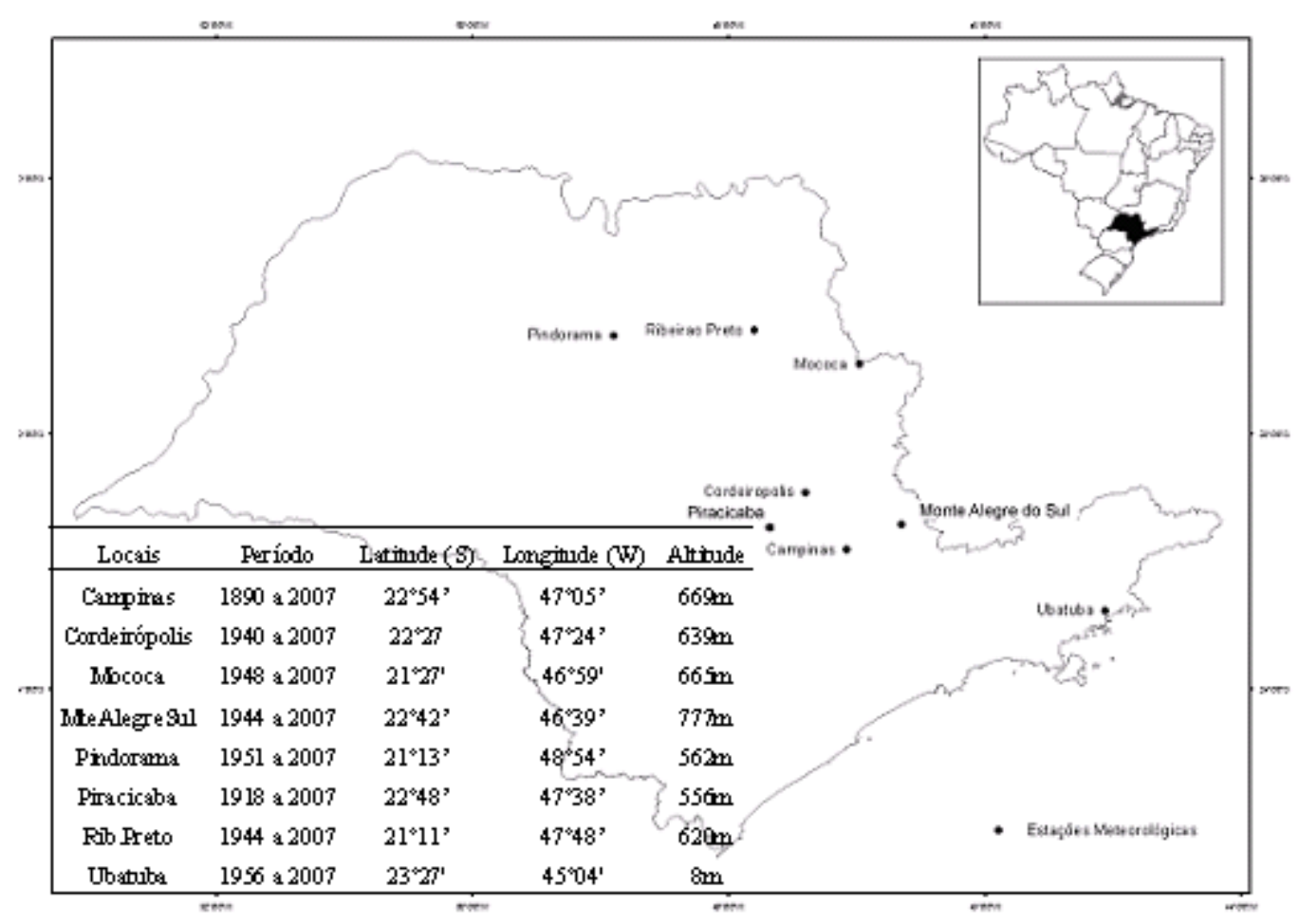

Figura 1 - Localidades e respectivas séries meteorológicas, pertencentes à Secretaria de Agricultura e Abastecimento do Estado de São Paulo, utilizadas no estudo. 
interessante alternativa para verificar se distintos períodos de uma série histórica podem ser considerados oriundos da mesma população teórica. $\mathrm{O}$ teste compara a verossimilhança associada a uma hipótese de nulidade (Ho) à verossimilhança associada a uma hipótese alternativa $\mathrm{H}_{\mathrm{A}}$. Ho considera que os distintos sub-períodos pertencem à mesma distribuição $\mathrm{e}$ é rejeitada, quando as verossimilhanças associadas à $\mathrm{H}_{\mathrm{A}}$ são suficientemente elevadas para que as diferenças estatísticas entre os sub-períodos sejam consideradas apenas variações amostrais. Uma interpretação relacionada é que a rejeição de Ho indica que a probabilidade paramétrica de ocorrência associada a um dado valor de Xt, difere significativamente (ao nível escolhido) entre as amostras. Ho é rejeitado se o valor de $\Lambda^{*}$ for superior a um dado quantil da distribuição qui-quadrado. $\mathrm{O} \Lambda *$ foi aplicado em todas as localidades considerando-se os sub-períodos utilizados nos teste te F.

A primeira etapa para a interpretação dos resultados do $\Lambda^{*}$, é baseada na determinação do valor crítico que estabelece a significância do teste. Entretanto, não existem na literatura, trabalhos que tenham analisado tal patamar para as condições de temperatura do ar no Estado de São Paulo. A fim de contornar esse problema, foi calculado o valor "p", que representa a probabilidade específica de ocorrência do valor calculado do teste estatístico e dos demais valores possíveis desse teste que seriam desfavoráveis à Ho. Em outras palavras, na condução do $\Lambda^{*}$, Ho não é rejeitado até o valor "p de significância". Quanto mais distintos os parâmetros estatísticos de cada sub-período, maior o valor de $\Lambda^{*}$ e menor o valor $\mathrm{p}$ de significância.

Apesar de sua robustez, o teste $\Lambda^{*}$ analisa apenas a probabilidade de ocorrência associada a cada valor de Tmax dentro das amostras. A distribuição temporal (seqüência) dos valores não é analisada. Esse tipo de análise pode ser realizado por meio dos métodos não paramétricos.

\section{2 Métodos não paramétricos}

Os métodos não paramétricos utilizados foram o teste de Mann-Kendall (MK), e de Pettitt. Inicialmente proposto por Mann (1945) e Kendall e Stuart (1967), o teste não paramétrico MK pode ser definido, para uma série $X_{t}=\left\{X_{1}, X_{2}, \ldots X_{i}, X_{j}, \ldots \ldots\right.$ $\mathrm{X}_{\mathrm{n}}$ ) por:

$$
\begin{aligned}
& \mathrm{T}=\sum_{\mathrm{j}<\mathrm{i}} \operatorname{sinal}\left(\mathrm{X}_{\mathrm{i}}-X_{\mathrm{j}}\right) \text { em que: } \\
& \operatorname{sinal}\left(\mathrm{X}_{\mathrm{i}}-\mathrm{X}_{\mathrm{j}}\right)=\left\{\begin{array}{l}
1 ; \text { para } \mathrm{X}_{\mathrm{i}}-\mathrm{X}_{\mathrm{j}}>0 \\
0 ; \text { para } \mathrm{X}_{\mathrm{i}}-\mathrm{X}_{\mathrm{j}}=0 \\
-1 ; \text { para } \mathrm{X}_{\mathrm{i}}-\mathrm{X}_{\mathrm{j}}<0
\end{array}\right\}
\end{aligned}
$$

considerando a inexistência de observações adjacentes iguais e a independência temporal (ruído branco) entre os $\mathrm{n}$ valores da série; quando não há presença de tendências $\mathrm{T}$ é normalmente com média $\mathrm{E}(\mathrm{T})=0$ e variância $\operatorname{Var}(\mathrm{T})$. $\mathrm{O}$ valor final da estatística MK pode ser estimado por:

$$
\mathrm{MK}=\left\{\begin{array}{l}
\frac{\mathrm{T}-1}{\sqrt{\operatorname{Var}(\mathrm{T})}} ; \text { para } \mathrm{T}>0 \\
0 ; \text { para } \mathrm{T}=0 \\
\frac{\mathrm{T}+1}{\sqrt{\operatorname{Var}(\mathrm{T})}} ; \text { para } \mathrm{T}<0
\end{array}\right\}
$$

em que: $\operatorname{Var}(\mathrm{T})=\frac{n(n-1)(2 n+5)}{18}$

Ho não é rejeitada se, em um teste bilateral, o valor absoluto do MK for menor que um determinado valor da distribuição normal reduzida. Autores como Marengo et al. (2007) utilizam o nível de significância $=0,05$. Adotando-se esse mesmo nível, Ho não será rejeitada sempre $-1,96<\mathrm{MK}<$ 1,96. Tendências de elevação ou queda resultam em valores positivos ou negativos de MK.

O teste de Pettitt (Pettitt, 1979) indica se duas amostras (no presente caso, oriundas da mesma série) $\mathrm{X}_{1}, \ldots, \mathrm{X}_{\mathrm{t}} \mathrm{e}$ $\mathrm{X}_{\mathrm{t}+1}, \ldots, \mathrm{X}_{\mathrm{T}}$ são da mesma população. A estatística Ut,T verifica o número de vezes que um elemento da primeira amostra é maior que um elemento da segunda. A estatística Ut,T é estimada pela Equação 3.

$$
U_{t, T}=U_{t-1, T} \sum_{j=1}^{T} \sin a l(X t-X j)
$$

$$
\text { pata } t=2 \ldots . \mathrm{T} \text { e } 1 \leq \mathrm{t} \leq \mathrm{T}
$$

Após essa etapa, a estatística $\mathrm{k}(\mathrm{t})$ assume o maior valor absoluto dos fatores Ut, $\mathrm{T}$; indicando o ponto aproximado da alteração nos valores da série (changing point). A significância de $\mathrm{k}(\mathrm{t})$ é dada pela Equação 4, em que o valor crítico desse fator é estimado por:

$$
k(t)_{\text {critico }}=\sqrt{\frac{\left(T^{3}+T^{2}\right)[-\ln (p / 2)]}{6}}
$$

Analisando-se as Equações 3 e 4, verifica-se que este teste é uma versão do teste de homogeniedade de populações de Mann-Whitney (teste U). Trabalhos como os de Victoria et 
al., (1998), Back (2001) e Mortatti (2004), adotam o nível de significância 5\% para a condução desse método.

Os métodos não paramétricos foram aplicados considerando: a) os anos de 1948/2007 nas localidades de Campinas, Cordeirópolis, Mococa, Monte Alegre do Sul, Piracicaba, Ribeirão Preto e Ubatuba; b) todo o período disponível nas localidades de Campinas, Pindorama e Ubatuba; c) os anos de 1917/2007 nas localidades de Campinas e Piracicaba. É importante ressaltar que, especialmente no período comum de 1948/2007, se os métodos não paramétricos detectarem tendências climáticas com significância e data inicial bastante distinta entre as localidades, as indicações de Dufek

Tabela 1 - Valores do teste Komogorov-Smirnov (KS) aplicados à análise de aderência das séries de temperatura máxima média anual (Tmax) à distribuição normal em oito localidades do Estado de São Paulo.

\begin{tabular}{lcccc}
\hline & Teste KS & \multicolumn{3}{c}{ Valor crítico } \\
\hline \multicolumn{1}{c}{ Local } & Tmax & $5 \%$ & $10 \%$ & $20 \%$ \\
\hline Campinas (1890 a 2007) & 0,095 & 0,126 & 0,112 & 0,099 \\
Cordeirópolis (1940 a 2007) & 0,045 & 0,166 & 0,148 & 0,130 \\
Mococa (1946 a 2007) & 0.019 & 0.177 & 0.158 & 0.138 \\
Mte Alegre do Sul (1944 a 2007) & 0,086 & 0,173 & 0,154 & 0,135 \\
Pindorama(1951 a 2007) & 0,059 & 0,181 & 0,162 & 0,142 \\
Piracicaba(1918 a 2007) & 0,099 & 0,144 & 0,129 & 0,113 \\
Ribeirão Preto (1943 a 2007) & 0,108 & 0,170 & 0,151 & 0,133 \\
Ubatuba(1956 a 2007) & 0.086 & 0.188 & 0.168 & 0.147 \\
\hline
\end{tabular}

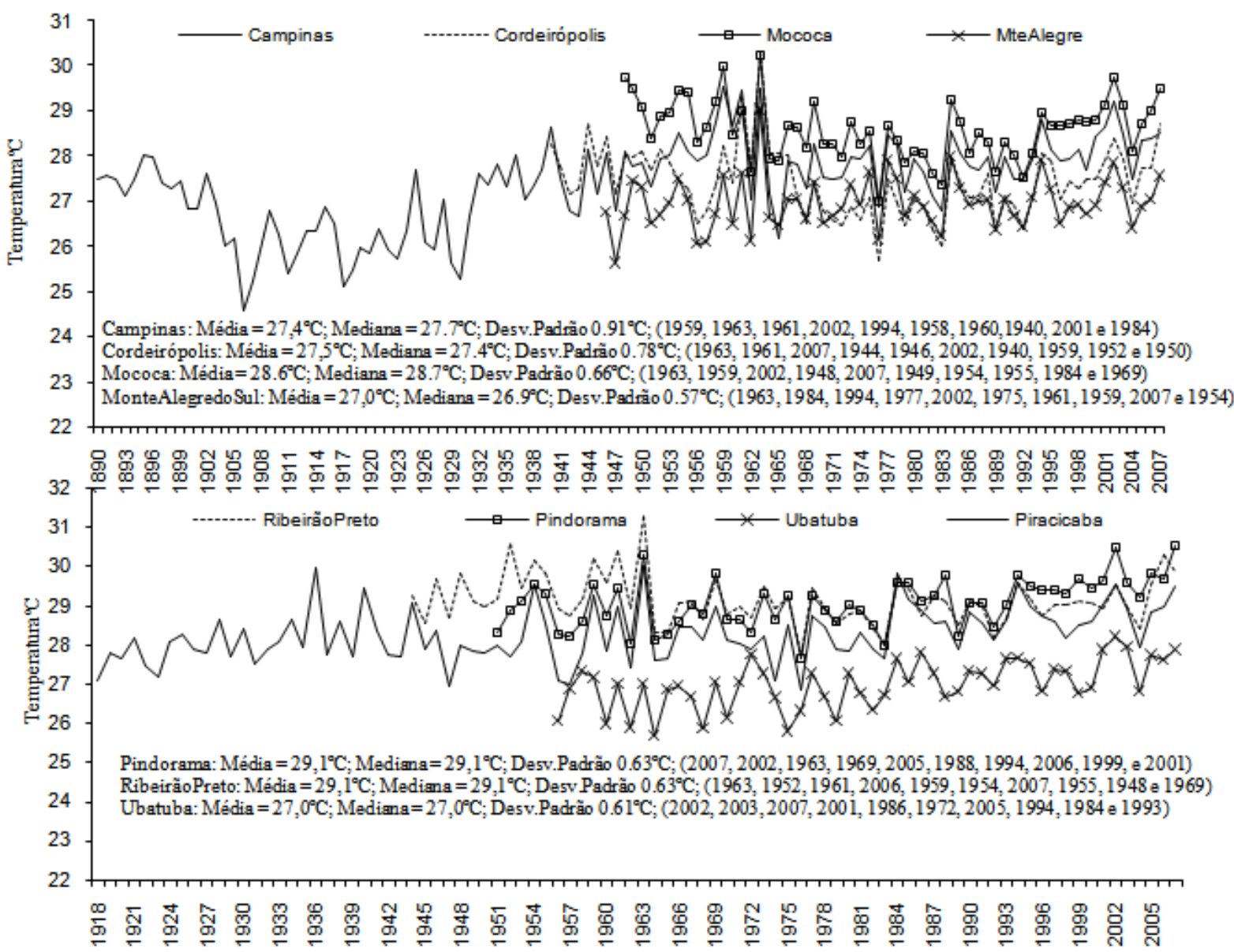

Figura 2 - Séries históricas de temperatura máxima média anual (Tmax) de oito localidades do Estado de São Paulo. Entre parentes o ano de ocorrência dos 10 maiores valores de cada série em ordem decrescente. 
e Ambrizzi (2006) e Blain et al. (2009), relativas à marcantes influências de fatores de escala local, tais como urbanização, na variabilidade temporal dos valores de Tmax do estado de São Paulo, tornam-se uma hipótese bastante provável.

\section{RESULTADOS E DISCUSSÃO}

Observa-se na Tabela 1, que todas as séries de Tmax podem ser consideradas oriundas de uma população com distribuição normal. Essa mesma característica pode ser observada em todos os sub-períodos utilizados em todas as localidades do estudo. Esse ajuste a um mesmo tipo de distribuição permite a aplicação dos testes t $\mathrm{e} F$. Ao contrário do observado por Blain et al. (2009) para as séries de temperatura mínima do ar em Campinas, não há na Figura 2, claras indicações de tendências climáticas nas séries de Tmax, tendo em vista a inexistência de picos máximos ou mínimos no ponto final das séries temporais.

Tabela 2 - Aplicação dos testes t e F para a série de temperatura máxima média anual das localidades de Campinas-SP e Piracicaba nos períodos de 1918 a 1947; 1948 a 1977 e 1978 a 2007.

\begin{tabular}{|c|c|c|c|c|c|c|}
\hline & \multicolumn{2}{|c|}{$\begin{array}{l}\text { Variâncias } \\
\text { equivalentes }\end{array}$} & \multicolumn{2}{|c|}{$\begin{array}{l}\text { Variâncias } \\
\text { diferentes }\end{array}$} & \multicolumn{2}{|c|}{$\begin{array}{l}\text { Variâncias } \\
\text { diferentes }\end{array}$} \\
\hline & $P 2$ & $P 1$ & $P 3$ & $P 2$ & $P 3$ & $P 1$ \\
\hline Média & 28.0 & 27.0 & 28.0 & 28.0 & 28.0 & 27.0 \\
\hline Variância & 0.61 & 0.94 & 0.27 & 0.61 & 0.27 & 0.94 \\
\hline $\mathrm{F}$ & 1,434 & & $1,981 *$ & & $2,853^{*}$ & \\
\hline F crítico uni-caudal & 1,861 & & 1,861 & & 1,861 & \\
\hline$t$ & $4.277^{*}$ & & 0.299 & & $5.061^{*}$ & \\
\hline \multirow[t]{4}{*}{ t crítico bi-caudal } & 2,002 & & 2,008 & & 2,008 & \\
\hline & \multicolumn{4}{|c|}{ Piracicaba-SP } & & \\
\hline & \multicolumn{2}{|c|}{$\begin{array}{l}\text { Variâncias } \\
\text { diferentes }\end{array}$} & \multicolumn{2}{|c|}{$\begin{array}{l}\text { Variâncias } \\
\text { diferentes }\end{array}$} & \multicolumn{2}{|c|}{$\begin{array}{l}\text { Variâncias } \\
\text { diferentes }\end{array}$} \\
\hline & $P 2$ & $P I$ & $P 3$ & $P 2$ & P3 & $P 1$ \\
\hline Média & 28,1 & 28,0 & 28.6 & 28.1 & 28.6 & 28,0 \\
\hline Variância & 0.55 & 0,42 & 0,31 & 0.55 & 0.31 & 0,42 \\
\hline $\mathrm{F}$ & 1,29 & & 1,74 & & 1,35 & \\
\hline F crítico uni-caudal & 1,861 & & 1,861 & & 1,861 & \\
\hline $\mathrm{t}$ & 0.36 & & $3,00 *$ & & $3.67 *$ & \\
\hline t crítico bi-caudal & 2.008 & & 2.008 & & 2.008 & \\
\hline
\end{tabular}

*Significativo à $5 \%$
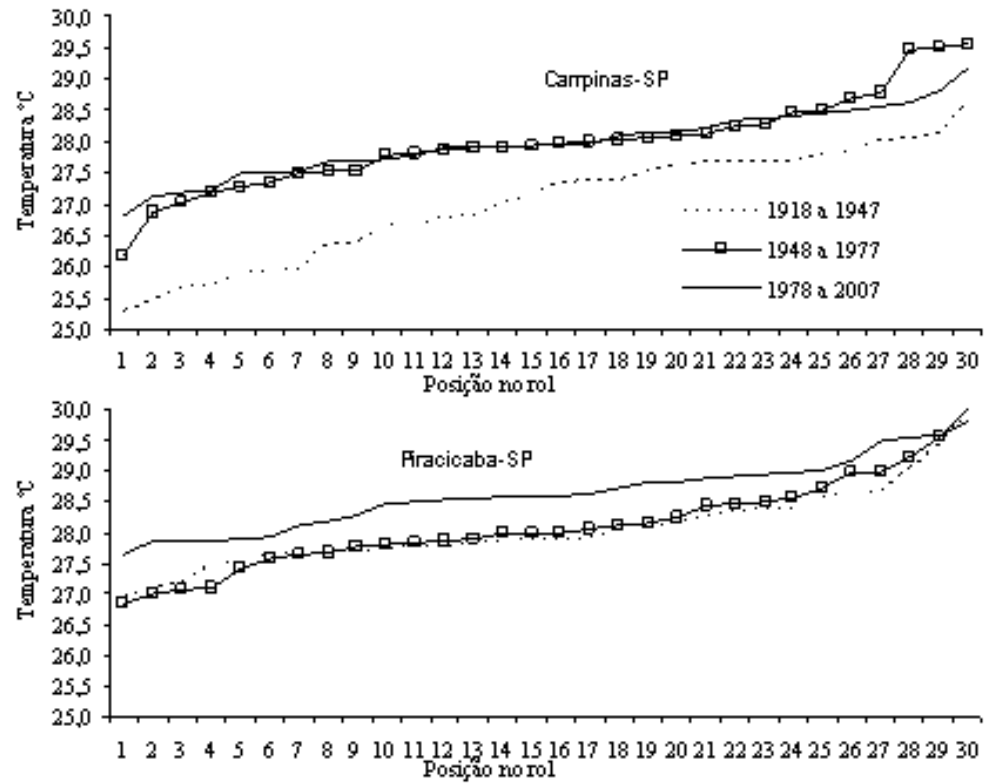

Figura 3 - Valores da temperatura máxima média anual, organizados em rol, relativos aos períodos de 1918/1947, 1948/1977 e 1978 a 2007 observados nas localidades de Campinas-SP e Piracicaba-SP. 
São ilustradas na Tabela 2 e na Figura 3 a aplicação dos testes $\mathrm{t}$ e $\mathrm{F}$ e os dados de Tmax, organizados em ordem crescente (rol), relativos aos períodos de 1918/1947; 1948/1977 e 1978/2007 para as localidades de Campinas e Piracicaba.

Analisando a Tabela 2 para a localidade de Campinas, nota-se que há diferença estatística ( $5 \%$ de significância) entre a média aritmética dos valores de Tmax relativos aos sub-períodos de 1918/1947 e 1948/1977; 1918/1947 e 1978/2007. Não houve diferença estatística entre os sub-períodos de 1948/1977 e 1978/ 2007. Em outro aspecto, nota-se que a variância da amostra 1978/2007 foi menor (diferença com nível 5\% de significância) do que as observadas nas amostras de 1918/1947 e 1948/1977. Nesse sentido, observando a Figura 3, nota-se que na amostra de 1948/1977 encontram-se os maiores valores de Tmax. Essa característica, aliada ao valor constante da média das amostras
1948/1977 e 1978/2007 e as observações realizadas com base na Figura 2, fornecem suporte à afirmação de que não há marcantes tendências climáticas na série de temperatura máxima anual do município de Campinas-SP. Na localidade de Piracicaba, todos os sub-períodos analisados apresentaram homocedasticidada das variâncias amostrais, contudo, de acordo com o teste thouve aumento médio significativo dos dados anuais de Tmax relativos aos últimos 30 anos dessa série. Na Figura 4 a aplicação dos testes t e F é ilustrada para as demais séries do estudo.

Analisando a Figura 4, nota-se elevação nos dados de Tmax, que resultou em médias aritméticas estatisticamente diferentes, nas localidades de Ubatuba e Pindorama. Nas localidades de Mococa e Monte Alegre do Sul, o teste t e o rol dos dados indicam que as magnitudes médias dos dados de Tmax observados nas duas amostras foram semelhantes. Nas
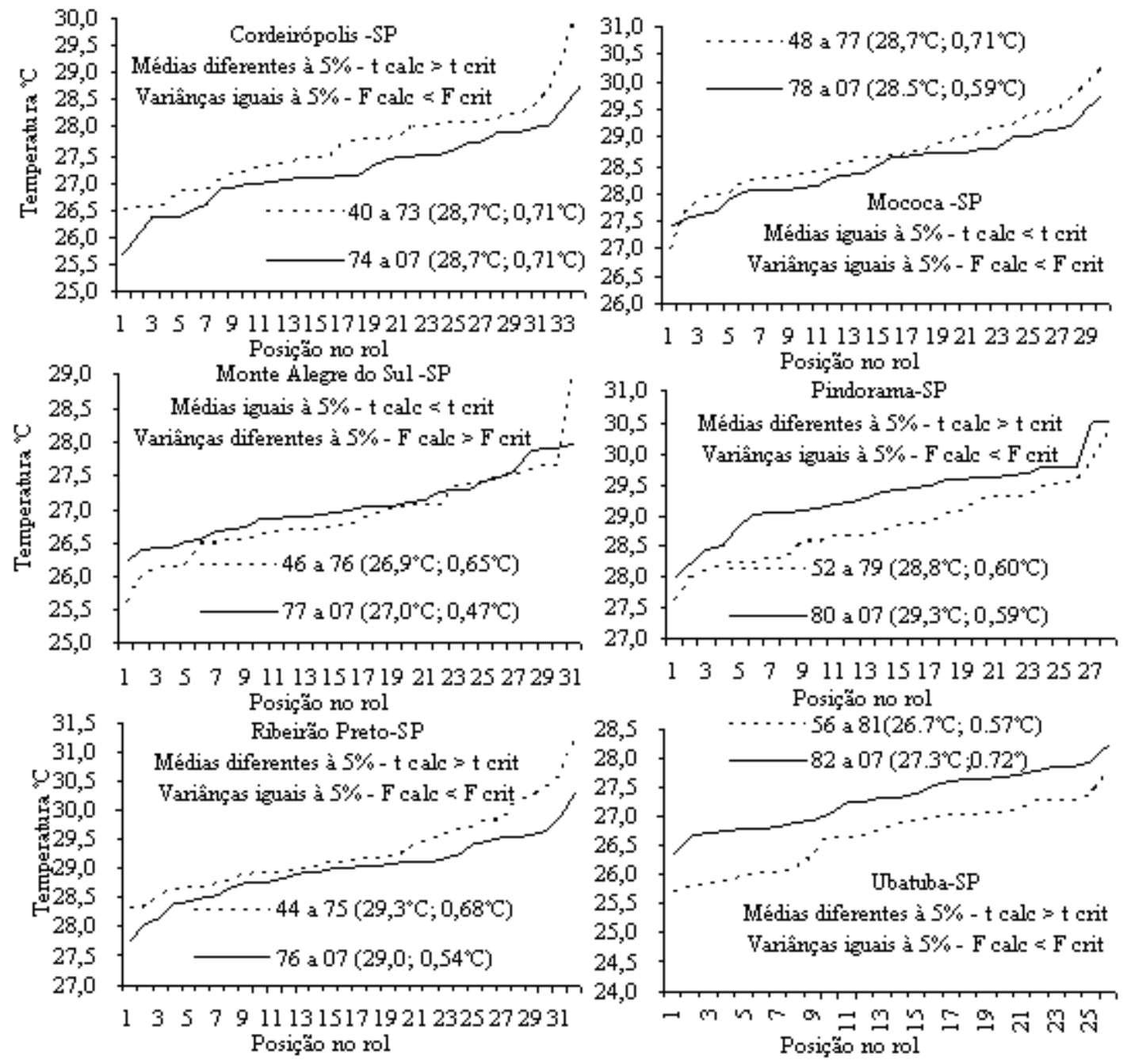

Figura 4 - Aplicação dos testes t e F para a série de temperatura máxima média anual das localidades de Cordeirópolis, Mococa, Monte Alegre do Sul, Pindorama, Ribeirão Preto e Ubatuba pertencentes ao Estado de São Paulo. Cada sub-período foi organizado em rol. 
localidades de Cordeirópolis e Ribeirão Preto observa-se queda nos valores dessa variável, quando são comparados os períodos mais antigos aos mais recentes. Em termos de variância, nas localidades de Monte Alegre do Sul e Ubatuba observou-se diferença significativa entre as amostras. Para a região de Monte Alegre do sul (onde houve redução na variância relativa aos anos de 1977 a 2007), essa diferença foi especialmente causada pela diferença nos extremos inferiores e superiores dos rol das amostras. Nessa localidade, os 5 menores valores de Tmax, observados entre 1946 e 2007 ocorreram no sub-período de $1946 / 1976$ e o maior valor dessa amostra $\left(29^{\circ} \mathrm{C}\right.$, ocorrido em 1963) é $1^{\circ} \mathrm{C}$ superior ao maior valor registrado no sub-período de 1977/2007 (ocorrido em 1984). A série relativa à localidade de Ubatuba foi a única a apresentar aumento no grau de dispersão de seus valores relativos aos anos mais recentes.

Observando a Figura 4, torna-se também interessante ressaltar a limitação associada ao tratamento do clima como sendo o valor médio das condições de tempo observadas em uma região e período (definição ainda utilizada em alguns métodos agrometeorológicos). Na localidade de Monte Alegre do Sul, as características opostas observadas nas posições extremas do rol relativo ao sub-período de 1946/1976 (Figura 4), produziram uma "compensação" no valor final da média aritmética da amostra considerada. Dessa forma, obteve-se um teste $\mathrm{t}$ não significativo entre os dois períodos analisados, apesar de terem ocorrido, de acordo com o teste $\mathrm{F}$, diferenças significativas nas variâncias relativas aos dois sub-períodos em que essa série foi sub-dividida. Com isso, torna-se evidente a necessidade de avaliar possíveis mudanças climáticas por meio de testes que considerem todos os valores presentes em cada amostra. Na Figura 5 é ilustrada a aplicação do $\Lambda^{*}$ nas séries de Campinas e Piracicaba.

Na Figura 5, verifica-se que a aplicação do $\Lambda^{*}$ nos três sub-períodos da série de Campinas, indica acentuada alteração na probabilidade de ocorrência associada a cada valor de cada amostra. Considerando-se a aplicação deste teste apenas nas amostras de 1948/1977 e de 1978/2007, bem como a representação gráfica de cada distribuição, pode-se afirmar, assim como indicado pelo teste $t$, que tal alteração ocorreu após o sub-período de 1918/1947. Comparando-se as formas das funções densidade de probabilidade (FDP) relativas às amostras de 1948/1977 e 1978/2007, fica evidente a queda na variância entre essas duas amostras (também indicada pelo teste F). O sub-período mais recente apresenta maior densidade de probabilidade de ocorrência dos valores Xt próximos à média aritmética da distribuição, e consequentemente, menor probabilidade de ocorrência dos valores extremos em ambas as caldas da distribuição. Na localidade de Piracicaba, também nota-se acentuada alteração na probabilidade de ocorrência associada a cada valor Xt entre as três amostras, sendo que tal alteração é principalmente atribuída à amostra de 1978/2007. Assim como na localidade de Campinas, a FDP dos últimos 30 anos apresenta o menor grau de dispersão. Entretanto, nota-se que a FDP da amostra de 1978/2007 em Piracicaba, quando comparada às demais FDP dessa série, apresenta forte deslocamento à direita atribuindo, com isso, maior probabilidade de ocorrência a valores mais elevados de Tmax anual. Comparando esses últimos resultados às indicações de Sansigolo (2008), descritas no item 1, observa-se que esse marcante deslocamento à direita nos valores anuais analisados, não resultou em elevação dos dados extremos diários de temperatura máxima em Piracicaba.

Sob o ponto de vista de consistência dessas séries, é importante ressaltar. que em ambas há um forte deslocamento à direita das FDP. Na série de Campinas, essa mudança brusca ocorre, de forma bastante acentuada, após o sub-período de 1918/1947. Apesar de utilizada em diversos estudos, tais como Blain e Brunini (2005) e Blain e Brunini (2007), é interessante
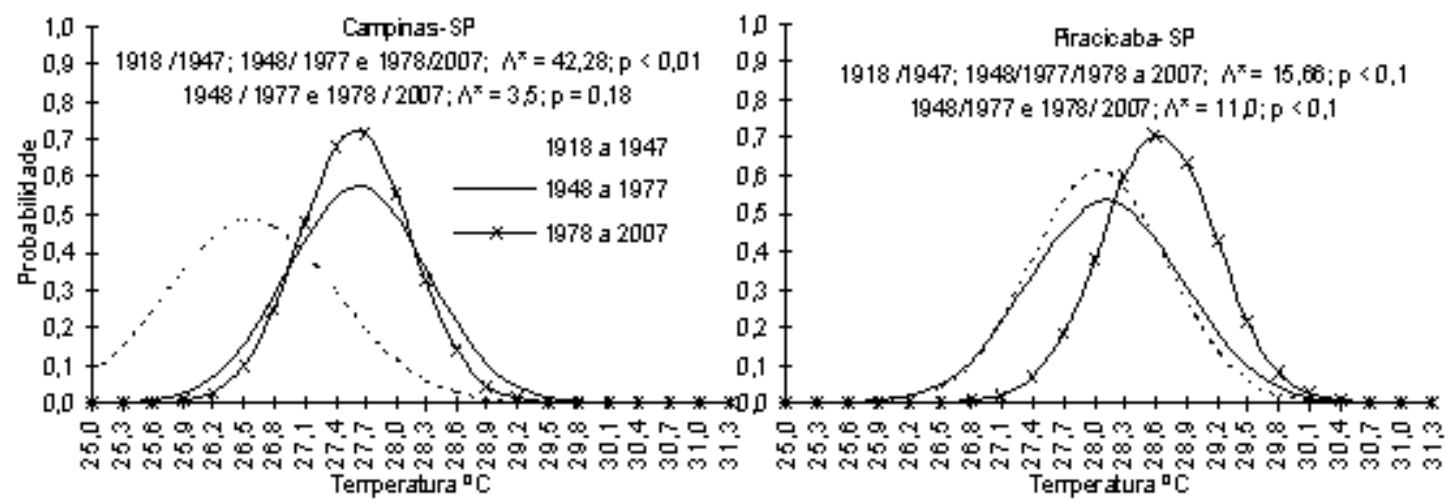

Figura 5 - Teste da razão da verossimilhança aplicado às séries de temperatura máxima média anual das localidades de Campinas e Piracicaba pertencente ao Estado de São Paulo. 
verificar que esse deslocamento não tenha sido salientado em nenhum artigo científico. Com isso, independente das razões ou forçantes físicas causadoras dessa característica, sua ilustração torna-se relevante. Na região de Piracicaba, essa alteração ocorre mais tardiamente, após a amostra de 1948/1977, e com menor intensidade. Considerando que o crescimento demográfico da localidade de Campinas ocorreu de forma mais precoce e intensa que na região de Piracicaba, a hipótese de que essas alterações são fortemente relacionadas a fatores de escala local (tais como urbanização) torna-se razoável. $\mathrm{O} \Lambda^{*}$ é ilustrado para as demais localidades na Figura 6

Na Figura 6, observa-se que nas séries de Pindorama e Ubatuba, as amostras relativas aos anos mais recentes (1979/2007 e 1982/2007, respectivamente), apresentam FDP com deslocamento à direita altamente significativo. A aplicação do teste $\Lambda^{*}$ nas localidades de Cordeirópolis e Ribeirão Preto indicou, com significância igual ou superior a
95\%, deslocamento à esquerda das FDP relativas aos anos mais recentes de cada série (1974/2007 e 1977/2007, respectivamente) e elevação da probabilidade de ocorrência de valores de Tmax próximos às médias aritméticas desses dois sub-períodos. $\mathrm{Na}$ região de Monte Alegre do Sul, comparando o sub-período de 1977/2007 ao de 1946/1976, observa-se aumento na freqüência de ocorrência de valores de Tmax próximos à média aritmética da distribuição, bem como um deslocamento à direita da calda esquerda (extremo inferior) da FDP. Entretanto, de acordo com o teste $\Lambda^{*}$, a significância dessa alteração é inferior a $90 \%$. A localidade de Mococa apresentou deslocamento à esquerda da FDP relativa aos anos mais recentes de sua série. Contudo, essa alteração apresentou um nível de significância inferior a 75\%.

Assim como apontado por Vincent et al. (2005), comparando-se os resultados do teste $\Lambda^{*}$ nas oito localidades, não é possível verificar uma consistente tendência de elevação temporal nas séries de Tmax analisadas. Nesse sentido, as
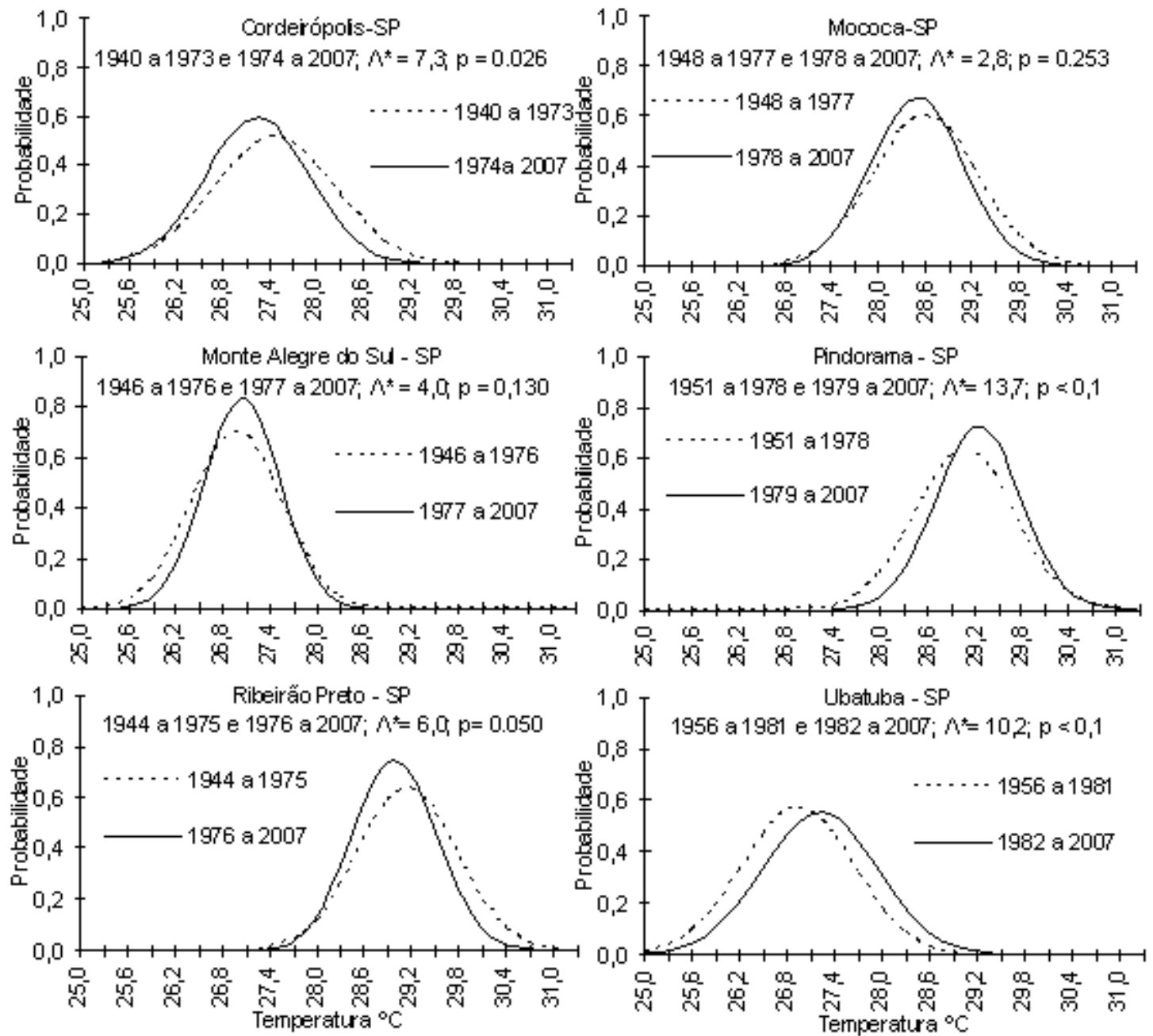

Figura 6 - Teste da razão da verossimilhança aplicado às séries de temperatura máxima média anual das localidades de Cordeirópolis, Mococa, Monte Alegre do Sul, Pindorama, Ribeirão Preto e Ubatuba pertencentes ao Estado de São Paulo. 
Tabela 3 - Aplicação dos testes t e F para a série de temperatura máxima média anual das localidades de Campinas-SP e Piracicaba nos períodos de 1918 a 1947; 1948 a 1977 e 1978 a 2007.

\begin{tabular}{|c|c|c|c|}
\hline Localidade & Período & MK & $\begin{array}{c}\text { Data aproximada } \\
\text { Pettitt }\end{array}$ \\
\hline Campinas & \multirow{6}{*}{1948 a 2007} & 0,94 & NS \\
\hline Cordeirópolis & & $-0,55$ & NS \\
\hline Mococa & & $-0,66$ & NS \\
\hline Monte Alegre Sul & & 1,01 & NS \\
\hline Piracicaba & & $3,60^{*}$ & 1983 \\
\hline Ribeirão Preto & & $-1,19$ & 1963 \\
\hline Pindorama & 1951 a 2007 & $3,66^{\star}$ & 1980 \\
\hline Ubatuba & 1955 a 2007 & $4,20^{*}$ & 1983 \\
\hline Campinas & \multirow{2}{*}{1917 a 2007} & $4,73^{*}$ & 1951 \\
\hline Piracicaba & & 4,40 * & 1983 \\
\hline Campinas & 1890 a 2007 & $6,17^{*}$ & 1943 \\
\hline
\end{tabular}

localidades de Piracicaba, Pindorama e Ubatuba apresentaram elevação ou deslocamento à direita nas FDP associadas aos valores de Tmax relativos aos anos mais recentes. Características opostas foram observadas nas séries de Cordeirópolis e Ribeirão preto. Nas séries de Monte Alegre do Sul e, especialmente, Mococa, não houve alterações significativas. Na Tabela 3 são ilustrados os resultados do emprego dos métodos não paramétricos.

Mesmo quando utilizado o período comum de 1948 a 2007, para a condução dos métodos não paramétricos, verificase, na Tabela 3, variabilidades temporais bastantes distintas nas séries de Tmax analisadas. Nesse período, apenas a série de Piracicaba apresentou valor significativo da estatística MK. Para os anos de 1917/2007, observa-se que tanto essa última localidade quanto a de Campinas apresentam teste MK significativo, indicando tendência de elevação nos dados de Tmax. Contudo, segundo o teste de Pettitt, a data inicial de elevação difere em aproximadamente 30 anos entre essas duas séries. Dessa forma, as informações da Tabela 3 parecem corroborar com as indicações de Dufek e Ambrizzi (2006) de que uma análise sobre o crescimento urbano nas cidades e as mudanças no uso do solo, assim como seus impactos no clima local, ainda se faz necessária, a fim de descrever em detalhes as diferenças gerais obtidas entre os resultados de cada estação meteorológica.

\section{CONCLUSÃO}

Nas localidades de Pindorama e Ubatuba, observase considerável elevação nos valores de Tmax ocorrida nos anos mais recentes dessas séries. Em contra partida, em Cordeirópolis, Mococa, Monte Alegre do Sul e Ribeirão Preto, verificou-se estabilidade ou até mesmo queda nos dados de Tmax relativos ao período mais recente dessas séries. Nas localidades de Campinas e Piracicaba nota-se marcantes deslocamentos à direita (elevação) das funções densidade de probabilidade associadas aos valores de Tmax. Em Campinas, essa alteração ocorreu de forma mais intensa e temporalmente anterior à de Piracicaba. Dessa forma, apesar da maioria das séries apresentar importantes variações em seus parâmetros estatísticos (variações climáticas), não foi possível estabelecer uma concomitância/significância na variabilidade temporal (elevação ou queda) nas oito séries de Tmax do Estado de São Paulo. Com isso, não houve detecção de tendências climáticas consistentes nos dados anuais de temperatura máxima do ar do Estado de São Paulo.

A função densidade de probabilidade, associada à distribuição normal, pode ser utilizada na descrição analítica das séries temporais de Tmax analisadas no presente trabalho.

\section{REFERÊNCIAS BIBLIOGRÁFICAS}

ALEXANDER, L.V.; ZHANG, X.; PETERSON, T.C.; CAESAR, J.; GLEASON, B.; TANK, A.M.G; HAYLOCK, M.; COLLINS, D.; TREVIN, B.; RAHIMZADEH, F.; TAGIPOU, A.; RUPA KUMAR, K.; REVADEKAR, J.; GRIFFITHS, G.; VINCENT, L.; STEPHENSON, D.; BURN, J.; AGUILLAR, E.; TAYLOR, M.; NEW, M.; ZHAI, P.; RUSTICUCCI, M.; VASQUEZ-AGUIRRE, J.L. Global observed changes in daily climate extremes of temperature 
and precipitation. Journal of Geophysical Research, Washington, v.111, 2006. Disponível em: <http://www. agu.org/journals/jd/jd0605/2005JD006290/>. Acesso em: 2 dez. 2008.

BACK, A.J. Aplicação de análise estatística para identificação de tendências climáticas. Pesquisa Agropecuária Brasileira, Brasília, v.36, p.717-726, 2001.

BLAIN, G.,C., BRUNINI, O., Avaliação e adaptação do Índice de Severidade de Seca de Palmer (PDSI) e do Índice Padronizado de Precipitação (SPI) às condições climáticas do Estado de São Paulo. Bragantia, v.64.,n.4,p. 605-705, Campinas, 2005

BLAIN, G.,C., BRUNINI, O., Análise comparativa dos índices de seca de Palmer, Palmer adaptado e Índice Padronizado de Precipitação no Estado de São Paulo, Revista Brasileira de Meteorologia, v.22.,n.1,p. 105-111, Santa Maria, 2007

BLAIN., G.,C., PICOLI, M., C., A., LULU, J., Análises estatísticas das tendências de elevação nas séries anuais de temperatura mínima do ar no Estado de São Paulo, Bragantia, v.68, n.3, 2009 (prelo)

DALE, R.F. Applied Climatogy. Indiana: Purdue University, USA, 1968. 125p.

DUFEK, A. S., AMBRIZZI, T., Variabilidade Climática da Temperatura no Estado de São Paulo. In: Congresso Brasileiro de Meteorologia 14., 2006, Florianópolis. Anais... Florianópolis: Sociedade Brasileira de Meteorologica, 2006

FREI, C.; SCHAR, C. Detection probability of trends in rare events: Theory and application to heavy precipitation in the Alpine region. Journal of Climate, Zurich, v.14, p.15681584, 2000.

HAYLOCK, M.R.; PETERSON, T.C.; ALVES, L.M.; AMBRIZZI, T.; ANUNCIAÇÃO, Y.M.T.; BAEZ, J.; BARROS, V.R.; BERLATO, M.A.; BIDEGAIN, M.; CORONEL, G.; CORRADI, V.; GARCIA, V.J.; GRIMM, A.M.; KAROLY, D.; MARENGO, J.A.; MARINO, M.B.; MONCUNILL, D.F.; NECHET, D.; QUINTANA, J.; REBELLO, E.; RUSTICUCCI, M.; SANTOS, J.L.; TREBEJO, I.; VINCENT L.A. Trends in total and extreme South American rainfall in 1960-2000 and links with sea surface temperature. Journal of Climate, Boston, v.19, p.1490-1512, 2006.

IPCC. The Physical Science Basis. Contribution of Working Group I to the Fourth Assessment Report of the Intergovernmental Panel on Climate Change. In: HOUGhTON, J.T. (Ed.). Climate Change. New York: Cambridge University Press, 2007.

KENDALL, M.A.; STUART, A. The advanced theory of statistics. Londres: Charles Griffin \& Company Limited, 1967. 690p.
MANN, H.B. Non-parametric tests against trend. Econometrica, Bristol, v.13, p.245-259, 1945.

MARENGO, J.; NOBRE, C.; RAIGOZA, D.; VALVERDE, M.; PISNITCHENKO, I.A.; OLIVEIRA, J.C.M. Boletim do Projeto: uso de cenários de mudanças climáticas regionais em estudos de vulnerabilidade e adaptação no Brasil e na América do Sul (GOF-UK-CPTEC), 2007. Disponível em: <www.cptec.inpe.br/mudancas_climaticas $>$. Acesso em: 8 dez. 2008.

MORTATTI, J., BORTOLETTO Jr., M., J., MILDE, L.,C.,E., PROSBST, J., Hidrologias dos rios Tietê e Piracicaba: Séries temporais de vazão e hidrogramas de cheia. Revista Ciência e Tecnológica. V.12, n.23, p.55-67. 2004

ORGANIZAÇÃO METEOROLÓGICA MUNDIAL. Climatic Change. Geneva, Switzerland: WMO, 1966. 80p.

PETTITT, A.N. A non-parametric approach to the change-point problem. Appl. Statis., v.28, n. 2, p.126-135, 1979.

SANSIGOLO, C.A. Distribuições de extremos de precipitação diária, temperatura máxima e mínima e velocidade do vento em Piracicaba, SP (1917-2006). Revista Brasileira de Meteorologia, São Paulo, v.23, n.3, p.341-346, 2008.

SANSIGOLO, C.A.; NERY, J.T. Distribuição de extremos de temperatura mínima no estado do Paraná. Revista Brasileira de Agrometeorologia, Santa Maria, v.8, n.2, p.247-253, 2000.

SCHICKEDANZ, P.T.; KRAUSE, G.F. A test for the scale parameters of two gamma distributions using the generalized likelihood ratio. Journal of applied meteorology, Boston, v.9, p.13-16, 1969.

VICTORIA, R., L., MARTINELLI, L., A., MORAES, J., M., BALlESTER, M., V., KRUSCHE, A., V., Surface air temperature variations in the Amazon Region and Its Borders during this century, Journal Of Climate, v.11, p. 1105-1110, 1998

VINCENT, L.A.; PETERSON, T.C.; BARROS, V.R.; MARINO, M.B.; RUSTICUCCI, M.; CARRASCO, G.; RAMIREZ, E.; ALVES, L.M.; AMBRIZZI, T.; BERLATO, M.A.; GRIMM, A.M.; MARENGO, J.A.; MOLION. L.; MONCUNILL, D.F.; REBELLO, E.; ANUNCIAÇÃO, Y.M.T.; QUINTANA, J.; SANTOS, J.L.; BAEZ, J.; CORONEL, G.; GARCIA, J.; TREBEJO, I.; BIDEGAIN, M.; HAYLOCK, M.R.; KAROLY, D. Observed trends in indices of daily temperature extremes in South America 1960-2000. Journal of Climate, Boston, v.18, p.50115023, 2005.

WILKS, D.S. Statistical methods in the atmospheric sciences. San Diego: Academic Press, $2^{\text {nd }}$ ed., 2006, 629p. 